\title{
ACTIVIDADES EXTRAESCOLARES DE LOS ADOLESCENTES ÚTILES PARA PROGRAMAS DE PREVENCIÓN DEL TABAQUISMO *
}

Equipo OCTOPUS: M. ${ }^{a}$ Luisa López González (1), Teresa López (2), Ángel Comas Fuentes (3), Pablo Herrero Puente (1), Javier González Blázquez (1), Antonio Cueto Espinar (1), Hywell Thomas (4), Jenny Douglas (4), Wolfan Markham (4), Anne CharIton (4), Hein de Vries (5), Ingrid Leijs (6), Ilse Mester (5) y Marlain Ausems (5).

(1) Facultad de Medicina. Área de Medicina Preventiva. Universidad de Oviedo.

(2) Universidad de Oviedo. Departamento de Estadística. Calvo Sotelo s/n. 33007 Oviedo.

(3) Centro de Salud de Otero. C/Otero s/n. 33008 Oviedo.

(4) Escuela de Educación de Birmingham. Reino Unido.

(5) Departamento de Educación para la Salud. Universidad de Maastricht. Países Bajos.

(6) Programa MUNDO de Educación para la Salud. Universidad de Maastricht. Países Bajos.

$\left({ }^{*}\right)$ Esta investigación ha sido subvencionada por el Programa «Europa contra el Cáncer» al equipo internacional OCTOPUS. Proyecto «Smoking Prevention and Children: Intervention in school and out of school», ref. SOC 94-202185.

\section{RESUMEN}

Fundamento: La prevalencia del tabaquismo en los adolescentes sigue indeseablemente elevada. Se precisan programas preventivos novedosos. Este estudio descriptivo transversal establece las variables conductuales asociadas al tabaquismo y las actividades más frecuentes de los adolescentes fuera de la escuela, útiles para plantear programas de prevención extraescolares.

Método: Los datos se recogieron con un cuestionario validado en una muestra representativa de escolares asturianos de 10-11 y 13-14 años. Mediante regresión logística se estudiaron las variables asociadas al consumo de tabaco.

Resultados: El porcentaje de inicio del consumo oscila entre el $14,5 \%$ y el $42,5 \%$, y el de fumadores regulares entre el $1,1 \%$ y el $12,4 \%$, respectivamente. Se construyeron 2 modelos con las variables asociadas significativamente a la conducta de fumar, los cuales clasifican correctamente en fumador/no fumador al $98,85 \%$ y $91,39 \%$ de los niños, según la edad. Las variables ambientales (accesibilidad al tabaco y al alcohol y frecuentación de lugares de riesgo) son las primeras integrantes del modelo. Las actividades extraescolares más frecuentes son: ver TV, leer y escuchar música y ver o practicar deporte.

Conclusión: Los resultados proporcionan claves para el diseño de programas extraescolares «a medida» de las actividades de los adolescentes: publicidad en TV y en soportes musicales (discos, cintas, etc.), e información escrita personalizada en el hogar, con mensajes transmitidos por líderes juveniles del deporte, la canción y la televisión

Palabras clave: Fumar. Adolescentes. Programas Preventivos. Tabaco.

\section{Correspondencia:}

Universidad de Oviedo.

Facultad de Medicina. Área de Medicina Preventiva.

Julián Clavería sin.

33006 Oviedo.

Correo electrónico: lopez@correo.uniovi.es.

\section{ABSTRACT}

\section{Teenager Extracurricular Activities Useful for Cigarette Smoking Habit Prevention Programs *}

Background: The cigarette smoking habit continues to be prevalent to a greater degree than would be desirable among teenagers. Innovative prevention programs are needed. This descriptive cross-sectional study sets out the behavior variables related to the cigarette smoking habit and the extracurricular activities in which teenagers are most frequently involved which ate useful for setting out extracurricular prevention programs.

Methods: The data was collected by means of a questionnaire validated in a representative sample of school age youths (ages 10-11 and 13-14) from Asturias. The variables entailed in cigarette smoking were analyzed using the regression method.

Results: The starting smokcr percentage is $14.5 \%-42.5 \%$, regular smokers totaling $1.1 \%$ and $12.4 \%$, respectively. Two models were constructed with the variables significantly related to smoking behavior, which are properly classified into smoker/non-smoker by $98.85 \%$ and $91.39 \%$ of the children, by ages. The environmental variables (availability of cigarettes and alcoholic beverages and regular visits to places entailing risk) are the major aspects comprising the model. The most common extracurricular activities are: watching TV, reading and listening to music and watching or playing sports.

Conclusion: The findings provide keys to planning extracurricular activities tailored to fit in with the activities most popular among teens: TV commercials and ads on music media (CD's, tapes, etc.) and printed information mailed directly to tecns at their homes, with messages conveycd by opinion-leaders among teens in the fields of sports, music and television.

Key words: Smoking. Teenagers. Prevention Programs. Cigarettes. 


\section{INTRODUCCIÓN}

Desde que el tabaquismo comenzó a ser considerado factor de riesgo para la salud, muchos países han implementado programas destinados a que las nuevas generaciones no empiecen a fumar. En España, aunque ha habido intentos de prevención mediante programas escolares, propiciados por la transversalidad del tema tabaco en los currículos escolares, tras promulgarse la LODE y la LOGSE, hay pocos programas de intervención educativa rigurosos y correctamente evaluados, y no parece que esté próxima la generación española sin tabaco. El Programa PASE ${ }^{1}$ encontró diferencias significativas en el consumo de tabaco entre el grupo de intervención y el de control en la evaluación a corto plazo, pero en ambos creció significativamente dicho consumo al año de la intervención².

Las últimas encuestas españolas de prevalencia $^{3-11}$ revelan frecuencias indeseables de consumo de tabaco en adolescentes, quc oscilan entre el $11,1 \%$ y el $55 \%$, dependiendo de la edad del encuestado, del año en que se realizó la encuesta y de diversas variables sociodemográficas.

Aunque el tabaquismo adolescente tiene una tendencia decreciente, según algunos estudios españoles ${ }^{4,8,12,13}$, los datos extranjeros no son consistentes: algunos autores señalan una tendencia alcista ${ }^{14-16}$ y otros demuestran disminución entre los chicos con aumento entre las chicas ${ }^{17.18}$. Además, en EEUU $^{18}$ se constató que la tendencia marcadamente decreciente entre 1974-80, la cual sufrió un estancamiento en los años 80 , probablemente debido al éxito de las campañas de promoción y publicidad del tabaco.

Si se confirma la tendencia decreciente de la frecuencia del tabaquismo adolescente en nuestro país, para mantenerla será necesario diseñar, implementar y evaluar programas preventivos, dirigidos a los niños y jóvenes. Dichos programas, si se desarrollan en el ámbito escolar, deberán elaborarse teniendo en cuenta los resultados obtenidos por otros análogos, durante más de 20 años de experiencia investigadora, en EEUU y el resto de Europa. En este sentido, casi toda la investigación previa ${ }^{19-31}$ coincide en que los programas más eficaces son aquéllos que se centran en el análisis de la presión social para fumar y en la adquisición de habilidades sociales para rechazar el tabaco. Pero, además, algunos autores y organismos $\mathrm{s}^{32-34}$ sugieren a futuros investigadores que los programas preventivos, además de intervenciones dentro de la escuela, diseñen y evalúen intervenciones educativas fuera de la misma, porque uno de los factores de riesgo de consumo de tabaco es cl fracaso escolar, y los adolescentes con mal expediente académico podrían rechazar cualquier actividad preventiva desarrollada en el ámbito del centro docente, precisamente por realizarse en él, ya que es vivido como impositivo y coercitivo por muchos de ellos.

Los objetivos de nuestro trabajo fueron:

- Conocer las actividades que concitan el interés de los niños fuera de la escuela, a fin de seleccionar las intervenciones preventivas extraescolares más adecuadas y el lugar o lugares en que tales intervenciones deberían aplicarse: intervención educativa «hecha a medida», según métodos actuales de Educación para la Salud (EpS) ${ }^{35,36}$.

- Comprobar si algunas de las variables, calificadas como determinantes de la conducta tabáquica en la literatura científica, se asocian significativamente con dicha conducta en los adolescentes y en qué medida lo hacen.

\section{MATERIAL Y MÉTODO}

Se trata de estudio epidemiológico descriptivo transversal, siendo la población estudiada 36.523 escolares asturianos de 10-11 años, repartidos en 1.415 aulas y 28.481 de 13-14 años procedentes de 1.012 aulas. Se realizó un muestreo aleatorio por conglomerados (unidad de selección el 
aula). La muestra fue estratificada por grupo de edad, lugar de residencia (municipios mayores y menores de 10.000 habitantes) y tipo de centro (público, privado y concertado). El tamaño muestral se calculó para una precisión del 3\% y un nivel de confianza del $95 \%$. No hubo pérdidas en los conglomerados. En total, en el curso 95-96 se encuestó a los alumnos de 55 aulas de $5 .^{\circ}$ y $6 .^{\circ}$ de EGB (1.479 alumnos de 10-11 años) y a los de 53 aulas de $7 .^{\circ}$ y $8 .^{\circ}$ de EGB y de $1 .^{\circ}$ de B.U.P. (1.516 alumnos de 13-14 años): $\mathrm{N}_{10-11}$ y $\mathrm{N}_{13-14}$ respectivamente, en adelante.

Para la recogida de la información, se elaboró un cuestionario con:

a) Variables socio-demográficas: edad, género, tipo de escuela y dincro disponible para gastar.

b) Variables relacionadas con algunos determinantes del tabaquismo, según la literatura científica: compra de tabaco, tabaquismo de convivientes y profesores y absentismo no justificado (hacer pira) - variables medidas de forma dicotómica (SI/NO) - y gusto por la escuela, ingesta de alcohol y rendimiento escolar - variables medidas mediante categorías ordinales-.

c) Variable para medir el consumo de tabaco, categorizada conforme se detalla en resultados (tabla 4).

d) Variables relativas a las actividades desarrolladas fuera del colegio, cuyo listado se elaboró con los resultados de una encuesta cualitativa realizada a 130 adolescentes del grupo diana:

Dentro de casa: ver tv/vídeo, usar ordenador, leer y oír música.

Fuera de casa: hacer deporte en un gimnasio o polideportivo, hacer deporte en un lugar no específico, acudir a un club o asociación cultural, hacer actividades propias de «scouts», pasear con la familia, callejear con los amigos, acudir al cine, acudir a un concierto, hacer compras, ver deporte, acu- dir a salas de juego (jugar con máquinas recreativas), acudir a una discoteca (incluidos locales sin alcohol para adolescentes), acudir a un bar (sólo para $\mathrm{N}_{13-14}$ ), acudir a casa de amigos, acudir a una asociacion escolar, acudir a una asociacion parroquial, tocar música con un grupo.

La inteligibilidad del cuestionario se comprobó mediante encuesta piloto a 150 niños de cada grupo. La estabilidad de lasrespuestas fue comprobada mediante test/re-test, en una submuestra aleatoria del $10 \%$ de la muestra, con un intervalo de 15 días. La encuesta fue realizada al grupo clase por un único encuestador -independiente y entrenado según protocolo establecido-, el cual dio instrucciones de cumplimentación y aclaró dudas en el momento de la encuesta.

La validez de construcción y contenido se sustenta en la encuesta cualitativa previa, en lo que concierne a las actividades, y la literatura científica para los determinantes del tabaquismo. La mayor parte de las variables no permiten la validez de criterio, por falta de uno externo de referencia accesible.

La consistencia interna de las respuestas dadas a las 3 actividades más practicadas por cada niño, se evaluó recogiendo el dato 2 veces en la misma encuesta, con preguntas distintas, y aplicando el Alfa de Cronbach a ambos datos.

Se realizó estadística descriptiva, análisis bivariable $\left(\mathrm{Ji}^{2}\right.$ para la significación estadística de las diferencias entre porcentajes) y análisis multivariantc mediante regresión logística, para determinar la importancia de las variables a estudio en la conducta de fumar, tanto esporádica como habitualmente: $0=$ NO FUMA (nunca ha fumado + Probó el tabaco + Antes fumaba pero ahora no); 1 $=$ FUMA (fuma $<1$ cigarrillo) por sem. + Fuma entre 1-6 cigarrillo por sem. + Fuma más de 6 cigarrillo por sem.). Todas las variables fueron previamente dicotomizadas, con excepción de la edad y el dinero dispo- 
nible. $\mathrm{El} 0=\mathrm{NO}$ presencia de la variable (no compra cigarrillos, no bebe alcohol, etc.) y el 1 = SI presencia de la variable (sí frecuenta discotecas, sí fuman sus profesores, etc.). Las excepciones a esta norma se recogen al pie de la tabla. Para la selección de las variables se utilizó el procedimiento «paso a paso", con un criterio de entrada de $\mathrm{p}=0,10$ y de salida de $\mathrm{p}=0,15$.

\section{RESULTADOS}

La estabilidad de las respuestas, excluidas las variables «edad» y «género», en las que el Indice de Kappa fue 1, osciló entre el 0,73 para el «dinero disponible» y el 0,94 para el tabaquismo de los convivientes. Y la consistencia interna para las tres actividades más frecuentes realizadas por cada niño varió entre el valor 0,84 y el 0,91 alfa de Cronbach.

La tabla 1 recoge los datos demográficos de la población a estudio. Un $85,7 \%$ de los $\mathrm{N}_{10-11}$ y un $88,9 \%$ de $\operatorname{los} \mathrm{N}_{13-14}$ reciben dinero
Tabla 1

Datos demográficos de los individuos participantes en la muestra

\begin{tabular}{|lccc|}
\hline Edad & $N$ & $\%$ & Género (\%) \\
\hline $10-11$ & 1.479 & 49,38 & $\begin{array}{l}\text { Chica }(51,3) \\
\text { Chico }(48,7)\end{array}$ \\
\hline $13-14$ & 1.516 & 50,62 & $\begin{array}{l}\text { Chica }(50,8) \\
\text { Chico }(49,2)\end{array}$ \\
\hline TOTAL & 2.995 & 100,00 & \\
\hline
\end{tabular}

para sus gastos: más de 500 pesetas por semana en el $13,3 \%$ y el $38,8 \%$ de los niños, respectivamente.

Las actividades más frecuentes dentro del hogar (tabla 2) son ver TV ó vídeo y leer. En los chicos mayores, la música desplaza a la lectura. El 96,4\% ve TV y el $87 \%$ lee, al menos, con frecuencia semanal, en el grupo $\mathrm{N}_{10-11}$. El 94,1\% ve TV y el $92,9 \%$ oye música, al menos con la frecuencia dicha, entre $\operatorname{los} \mathrm{N}_{13-14}$.

Actividades más frecuentes dentro de casa (n y \%)

\begin{tabular}{|c|c|c|c|c|c|c|}
\hline \multirow{2}{*}{ Actividad } & \multicolumn{6}{|c|}{ Frecuencia } \\
\hline & 6 & 5 & 4 & 3 & 2 & 1 \\
\hline \multicolumn{7}{|l|}{$T V$} \\
\hline $10-11$ & $1.168(79,0)$ & $216(14,6)$ & $42(2,8)$ & $11(0,7)$ & $4(0,3)$ & $38(2,6)$ \\
\hline $13-14$ & $1.206(79,6)$ & $214(14,1)$ & $61(0,4)$ & $11(0,7)$ & $4(0,3)$ & $20(1,3)$ \\
\hline \multicolumn{7}{|l|}{ Ordenador } \\
\hline $10-11$ & $167(11,3)$ & $308(20,8)$ & $227(15,3)$ & $123(8,3)$ & $121(8,2)$ & $533(36,0)$ \\
\hline $13-14$ & $112(7,4)$ & $261(17,2)$ & $241(15,9)$ & $139(9,2)$ & $152(10,0)$ & $611(40,3)$ \\
\hline \multicolumn{7}{|l|}{ Leer } \\
\hline $10-11$ & $756(51,1)$ & $350(23,7)$ & $180(12,2)$ & $49(3,3)$ & $38(2,6)$ & $106(7,2)$ \\
\hline $13-14$ & $559(36,9)$ & $418(27,6)$ & $244(16,1)$ & $112(7,4)$ & $74(4,9)$ & $109(7,2)$ \\
\hline \multicolumn{7}{|l|}{ Música } \\
\hline $10-11$ & $684(46,2)$ & $355(24,0)$ & $167(11,3)$ & $70(4,7)$ & $54(3,7)$ & $149(10,1)$ \\
\hline $13-14$ & $1.003(66,2)$ & $281(18,5)$ & $124(8,2)$ & $21(1,4)$ & $24(1,6)$ & $63(4,2)$ \\
\hline
\end{tabular}

$6=$ Casi todos los dias; $5=2-3 \mathrm{veces} / \mathrm{sem} ; 4=$ Aproximadantente $1 \mathrm{vez} / \mathrm{sem} ; 3=$ Al menos $1 \mathrm{vez} / \mathrm{mes} ; 2=$ Unas pocas veces al año $; 1-$ Nunca 0 casi nunca 
Fuera de la casa (tabla 3), el deporte - como practicante o como espectador-, pasear con la familia, hacer compras y callejear con los amigos, son las actividades más practicadas por los pequeños. Los de 13-14 años, además del deporte, que es la actividad que más interés concita, van más a casa de sus amigos y menos de compras, y disminuyen la frecuencia de paseos con la familia.

Tabla 3

Actividades más frecuentes fuera de casa (n y \%)

\begin{tabular}{|c|c|c|c|c|c|c|}
\hline \multirow{2}{*}{ Actividad } & \multicolumn{6}{|c|}{ Frecuencia } \\
\hline & 6 & 5 & 4 & 3 & 2 & 1 \\
\hline \multicolumn{7}{|l|}{ Gimnasio } \\
\hline $10-11$ & $170(11,5)$ & $337(22,8)$ & $110(7,4)$ & $55(3,7)$ & $72(4,9)$ & $735(49,7)$ \\
\hline $13-14$ & $142(9,4)$ & $305(20,1)$ & $155(10,2)$ & $85(5,6)$ & $158(10,4)$ & $671(44,3)$ \\
\hline \multicolumn{7}{|l|}{ Deporte } \\
\hline $10-11$ & $881(59,6)$ & $331(22,4)$ & $104(7,0)$ & $26(1,8)$ & $32(2,2)$ & $105(7,1)$ \\
\hline $13-14$ & $589(38,9)$ & $432(28,5)$ & $186(12,3)$ & $68(4,5)$ & $66(4,4)$ & $175(11,5)$ \\
\hline \multicolumn{7}{|c|}{ Club Cultural } \\
\hline $10-11$ & $31(2,1)$ & $33(2,2)$ & $45(3,0)$ & $23(1,6)$ & $37(2,5)$ & $1.310(88,6)$ \\
\hline $13-14$ & $54(3,6)$ & $74(4,9)$ & $162(10,7)$ & $70(4,6)$ & $102(6,7)$ & $1.054(69,5)$ \\
\hline \multicolumn{7}{|l|}{ Scouts } \\
\hline $10-11$ & $18(1,2)$ & $17(1,1)$ & $33(2,2)$ & $30(2,0)$ & $93(6,3)$ & $1.288(87,1)$ \\
\hline $13-14$ & $36(2,4)$ & $32(2,1)$ & $64(4,2)$ & $37(2,4)$ & $90(5,9)$ & $1.257(82,9)$ \\
\hline \multicolumn{7}{|l|}{ Familia } \\
\hline $10-11$ & $661(44,7)$ & $425(28,7)$ & $193(13,0)$ & $76(5,1)$ & $54(3,7)$ & $70(4,7)$ \\
\hline $13-14$ & $232(15,3)$ & $285(18,8)$ & $419(27,6)$ & $180(11,9)$ & $133(8,8)$ & $267(17,6)$ \\
\hline \multicolumn{7}{|l|}{ Caltejear } \\
\hline $10-11$ & $671(45,4)$ & $282(19,1)$ & $131(8,9)$ & $67(4,5)$ & $73(4,9)$ & $255(17,2)$ \\
\hline $13-14$ & $723(47,7)$ & $356(23,5)$ & $217(14,3)$ & $55(3,6)$ & $56(3,7)$ & $109(7,2)$ \\
\hline \multicolumn{7}{|l|}{ Cine } \\
\hline $10-11$ & $24(1,6)$ & $48(3,2)$ & $147(9,9)$ & $400(27,0)$ & $471(31,8)$ & $389(26.3)$ \\
\hline $13-14$ & $18(1,2)$ & $23(1,5)$ & $239(15,8)$ & $543(35,8)$ & $395(26,1)$ & $298(19,7)$ \\
\hline \multicolumn{7}{|l|}{ Concierto } \\
\hline $10-11$ & $8(0,5)$ & $8(0,5)$ & $13(0,9)$ & $46(3,1)$ & $183(12,4)$ & $1.221(82,6)$ \\
\hline $13-14$ & $9(0,6)$ & $2(0,1)$ & $6(0,4)$ & $53(3,5)$ & $321(21,2)$ & $1.125(74,2)$ \\
\hline \multicolumn{7}{|l|}{ Compras } \\
\hline $10-11$ & $547(37,0)$ & $377(25,5)$ & $262(17,7)$ & $151(10,2)$ & $70(4,7)$ & $72(4,9)$ \\
\hline $13-14$ & $200(13,2)$ & $241(15,9)$ & $311(20,5)$ & $446(29,4)$ & $188(12,4)$ & $130(8,6)$ \\
\hline \multicolumn{7}{|c|}{ Ver Deporte } \\
\hline $10-11$ & $553(37,4)$ & $316(21,4)$ & $212(14,3)$ & $105(7,1)$ & $93(6,3)$ & $200(13,5)$ \\
\hline $13-14$ & $365(24,1)$ & $307(20,3)$ & $301(19,9)$ & $160(10,6)$ & $168(11,1)$ & $215(14,2)$ \\
\hline \multicolumn{7}{|c|}{ Salas juego } \\
\hline $10-11$ & $82(5,5)$ & $121(8,2)$ & $123(8,3)$ & $114(7,7)$ & $95(6,4)$ & $944(63,8)$ \\
\hline $13-14$ & $67(4,4)$ & $149(9,8)$ & $243(16,0)$ & $150(9,9)$ & $167(11,0)$ & $740(48,8)$ \\
\hline
\end{tabular}


Tabla 3 (continuación)

\begin{tabular}{|c|c|c|c|c|c|c|}
\hline \multirow{2}{*}{ Actividad } & \multicolumn{6}{|c|}{ Frecuencia } \\
\hline & 6 & 5 & 4 & 3 & 2 & 1 \\
\hline \multicolumn{7}{|l|}{ Discoteca } \\
\hline $10-11$ & $14(0,9)$ & $5(0,3)$ & $19(1,3)$ & $18(1,2)$ & $130(8,8)$ & $1.293(87,4)$ \\
\hline $13-14$ & $20(1,3)$ & $51(3,4)$ & $164(10.8)$ & $78(5,1)$ & $174(11,5)$ & $1.029(67,9)$ \\
\hline \multicolumn{7}{|l|}{ Bar } \\
\hline $13-14$ & $88(5,8)$ & $148(9,8)$ & $235(15,5)$ & $129(8,5)$ & $150(9,9)$ & $766(50,5)$ \\
\hline \multicolumn{7}{|c|}{ Casa amigos } \\
\hline $10-11$ & $347(23,5)$ & $327(22,1)$ & $294(19,9)$ & $183(12,4)$ & $169(11,4)$ & $159(10,8)$ \\
\hline $13-14$ & $234(15,4)$ & $341(22,5)$ & $337(22,2)$ & $251(16,6)$ & $191(12,6)$ & $162(10,7)$ \\
\hline \multicolumn{7}{|c|}{ Asoc. escolar } \\
\hline $10-11$ & $75(5,1)$ & $94(6,4)$ & $56(3,8)$ & $16(1,1)$ & $21(1,4)$ & $1.217(82,3)$ \\
\hline $13-14$ & $18(1,2)$ & $33(2,2)$ & $75(4,9)$ & $10(0,7)$ & $20(1,3)$ & $1.360(89,7)$ \\
\hline \multicolumn{7}{|l|}{$\begin{array}{l}\text { Asociación } \\
\text { parroquial }\end{array}$} \\
\hline $10 m 11$ & $38(2,6)$ & $49(3,3)$ & $159(10,8)$ & $30(2,0)$ & $52(3,5)$ & $1.151(77,8)$ \\
\hline $13-14$ & $11(0,7)$ & $15(1,0)$ & $148(9,8)$ & $32(2,1)$ & $52(3,4)$ & $1.258(83,0)$ \\
\hline \multicolumn{7}{|c|}{ Tocar música } \\
\hline $10-11$ & $31(2,1)$ & $35(2,4)$ & $35(2,4)$ & $10(0,7)$ & $26(1,8)$ & $1.342(90,7)$ \\
\hline $13-14$ & $12(0,8)$ & $16(1,1)$ & $31(2,0)$ & $11(0,7)$ & $28(1,8)$ & $1.418(93,5)$ \\
\hline
\end{tabular}

$6=$ Casi todos $\operatorname{los}$ días $; 5=2-3$ veces $/ \mathrm{sem} ; 4=$ Aproximadamente 1 vez $/ \mathrm{sem} ; 3=$ Al menos 1 vez $/$ mes $; 2=$ Unas pocas veces al año; $1=$ Nunca o casi nunca.

Beben alcohol con frecuencia al menos semanal el $32,3 \%$ de los $\mathrm{N}_{10-11}$ y el $30,2 \%$ de los $\mathrm{N}_{13-14}$ (tabla 4).

En relación con el tabaco cabe destacar que, de los $\mathrm{N}_{10-11}: 2,8 \%$ ha comprado tabaco, $71,3 \%$ convive con fumadores y $62,3 \%$ ve fumar a sus profesores. $Y$ entre $\operatorname{los} \mathrm{N}_{13-14}$, es- tos porcentajes son $20,2 \%, 70,8 \%$ y $72,1 \%$, respectivamente.

La tabla 4 recoge también la frecuencia de consumo de tabaco pormenorizada: 14,5 de $\operatorname{los} N_{10-11}$ y $42,5 \%$ de los $N_{13-14}$ ha tenido contacto con el tabaco. Fuma con frecuencia al menos semanal el $1,1 \%$ y $12,4 \%$ respectivamente.

Tabla 4

Consumo de alcohol y tabaco (n y \%)

\begin{tabular}{|c|c|c|c|c|c|c|}
\hline \multirow[b]{2}{*}{ Edad } & \multicolumn{6}{|c|}{ Frecuencia consumo de alcohol } \\
\hline & $\begin{array}{c}\text { Más de } 6 \text { veces } \\
\text { por semana }\end{array}$ & $\begin{array}{l}\text { Entre } 2 \text { y } 6 \text { ve- } \\
\text { ces por semana }\end{array}$ & $\begin{array}{c}\text { No más de I vez } \\
\text { por semana }\end{array}$ & $\begin{array}{l}\text { Bebia antes, } \\
\text { ahora no bebo }\end{array}$ & $\begin{array}{l}\text { He probado en } \\
\text { alguna ocasión }\end{array}$ & Nunca \\
\hline $10-11$ & $3(0,2)$ & $24(1,6)$ & $451(30,5)$ & $13(0,9)$ & $459(31,0)$ & $529(35,8)$ \\
\hline \multirow[t]{3}{*}{$13-14$} & $10(0,7)$ & $48(3,2)$ & $399(26,3)$ & $28(1,8)$ & $627(41,4)$ & $404(26,6)$ \\
\hline & \multicolumn{6}{|c|}{ Frecuencia consumo de tabaco } \\
\hline & $\begin{array}{c}\text { Más de } 6 \text { veces } \\
\text { por semana }\end{array}$ & $\begin{array}{l}\text { Entre } 2 \text { y } 6 \text { ve- } \\
\text { ces por semana }\end{array}$ & $\begin{array}{c}\text { No más de l vez } \\
\text { por semana }\end{array}$ & $\begin{array}{l}\text { Fumaba antes, } \\
\text { ahora no fumo }\end{array}$ & $\begin{array}{l}\text { He probado en } \\
\text { alguna ocasión }\end{array}$ & Nunca \\
\hline $10-11$ & $1(0,1)$ & $2(0,1)$ & $13(0,9)$ & $14(0,9)$ & $184(12,5)$ & $1.265(85,5)$ \\
\hline $13-14$ & $46(3,0)$ & $44(2,9)$ & $99(6,5)$ & $99(6,5)$ & $357(23,6)$ & $871(57,5)$ \\
\hline
\end{tabular}


La autoevaluación del rendimiento escolar, el gusto por la escuela y el absentismo escolar injustificado se distribuyeron como sigue: Entre los $\mathrm{N}_{10-11}$ el 19,8\% consideraba su rendimiento entre regular y muy malo, al $27,3 \%$ la escuela le gustaba poco o nada y el $7,4 \%$ había hecho pira; entre $\operatorname{los} \mathrm{N}_{13-14}$ estos porcentajes fueron: $47,5 \%, 53,6 \%$ y $16,5 \%$.

$\mathrm{El}$ análisis bivariable intragrupos encuentra que fuman más los chicos que las chicas en el grupo $\mathrm{N}_{10-11}\left(\mathrm{Ji}^{2}\right.$ de Pearson $=6,86753$; $\mathrm{p}=0,008$ ). En el $\mathrm{N}_{13-14}$ no se hallaron diferencias significativas entre fumadores, ligadas al género $\left(\mathrm{Ji}^{2}=0,38772 ; \mathrm{p}=0,533\right)$, pero sí a la edad $\left(\mathrm{Ji}^{2}=65,9916 ; \mathrm{p}<0,0001\right)$, pues fuman significativamente más los de 14 que los de 13 años.

Finalmente, el análisis multivariante (tabla 5) muestra las variables que se asocian significativamente a la conducta de fumar. El modelo del grupo $\mathrm{N}_{10-11}$ clasifica correctamente en fumador / no fumador al $98,85 \%$ y el del grupo $\mathrm{N}_{13-14}$ al $91,39 \%$ de los estudiantes. Los modelos obtenidos difieren significativamente en su verosimilitud del modelo que incluye sólo la constante $(\mathrm{p}<0,016$ y $p<0,013$ para $N_{10-11}$ y $N_{13-14}$, respectivamente).

Tabla 5

Resultados del análisis multivariante mediante regresión logística

\begin{tabular}{|c|c|c|c|c|c|c|}
\hline $\begin{array}{c}\text { Orden de Selección } \\
\text { de las variables }\end{array}$ & Variables & Coeficiente & $p$ & Error Estandar & $O . R$ & I.C. \\
\hline \multicolumn{7}{|l|}{$(10-11)$} \\
\hline 1 & Comprar cigarrillos & 2,798 & 0,0001 & 0,626 & 16,4 & $4.81-56,0$ \\
\hline 2 & Beber alcohol & 2,518 & 0,0043 & 0,779 & 12,4 & $2.69-57,2$ \\
\hline 3 & Frecuentar discolecas & 1,696 & 0,0236 & 0,654 & 5,4 & $1.51-19,7$ \\
\hline \multirow[t]{2}{*}{4} & Estar en salas de juego & 1,354 & 0,0341 & 0,584 & 3,8 & $1,23-12,2$ \\
\hline & CONSTANTE & $-7,366$ & $<0,0001$ & 0,857 & & \\
\hline \multicolumn{7}{|l|}{$(13-14)$} \\
\hline 1 & Comprar cigarrillos & 3,295 & 0,0001 & 0,234 & 27,0 & $17,1-42,7$ \\
\hline 2 & Beber alcohol & 0,8764 & 0,0001 & 0,222 & 2,4 & $1,5-3,7$ \\
\hline 3 & Frecuentar discotecas & 0,5216 & 0,0025 & 0,235 & 1,6 & $1,06-2,67$ \\
\hline 4 & Hacer «pira» ${ }^{(a)}$ & $-0,6545$ & 0,0013 & 0,232 & 0,52 & $0,33-0,81$ \\
\hline 5 & Edad & 0,3812 & 0,0080 & 0,168 & 1,46 & $1,05-2,04$ \\
\hline 6 & Profesores fumadores & 0,7051 & 0,0095 & 0,271 & 2,02 & $1,19-3,45$ \\
\hline 7 & $\operatorname{Sexo}^{(b)}$ & $-0,4297$ & 0,0343 & 0,216 & 0,65 & $0,42-0,99$ \\
\hline \multirow[t]{2}{*}{8} & Dinero disponible & 0.1465 & 0,0496 & $0,81 \times 10^{-1}$ & 1,16 & $0,98-1,36$ \\
\hline & CONSTANTE & $-9,413$ & $<0,0001$ & 2,28 & & \\
\hline
\end{tabular}

(a) $0=$ Hace pira; $1=$ No hace pira. ${ }^{\text {(b) }} 0=$ Chica; $1=$ Chico

\section{DISCUSIÓN}

El porcentaje de los alumnos $\mathrm{N}_{10-11}$ escolarizados que nunca ha fumado $(85,5 \%)$ es muy parecido al encontrado por Villalbi ${ }^{3}$ entre los escolares de Barcelona en el curso 86-87 y en ambos estudios las chicas fuman menos que los chicos a estas edades. Sin embargo, en el grupo $\mathrm{N}_{13-14}$ nuestros porcentajes difieren: $57,5 \%$ de los asturianos aún no ha probado el tabaco, mientras que entre el 11 y el $15 \%$ de los barceloneses no lo ha hecho. En los dos estudios han desaparecido las diferencias ligadas al género entre los 
mayores. Sin embargo, nos aproximamos en la frecuencia de consumo semanal, con un $1,1 \%$ en $\mathrm{N}_{10-11}$ y un $12,4 \%$ en $\mathrm{N}_{13-14}$, ligeramente más bajos que los porcentajes de los niños barccloneses. Las diferencias podrían deberse a las características sociodemográficas de la población a estudio: niños de una gran metrópoli, tradicionalmente de alto riesgo tabáquico, frente a una muestra representativa de la comunidad autónoma asturiana, con participación rural, donde el control social de las conductas de riesgo es alto. En ambos estudios se comprueba que la experimentación con el tabaco aumenta de forma notable en el segundo grupo. No obstante, una limitación de nuestro trabajo es la falta de criterio externo (determinación de $\mathrm{CO}$ o de cotinina) que ratifique las frecuencias encontradas por encuesta, aunque la estricta confidencialidad de las encuestas había sido garantizada. No nos fue posible su utilización por falta de recursos.

Otro estudio ${ }^{13}$, cuyos datos se recogieron sólo un año antes que los nuestros, establece que el $43,4 \%$ de los niños catalanes de 14 años había fumado alguna vez: el porcentaje complementario $(56,7 \%)$ coincide con el de los niños asturianos de la misma edad que nunca había fumado.

Parece que los niños experimentan con el tabaco más precozmente que las niñas, pero en el grupo de 13-14 años las frecuencias de consumo se igualan, y es posible que en años posteriores las chicas fumen más que los chicos, como se ha puesto de manifiesto en otros estudios para grupos de edad más avanzados $6,9,17,37$. De hecho, nuestro análisis multivariante indica que ser mujer incrementa la probabilidad de fumar en el grupo de 13-14 años.

Los años transcurridos entre los estudios citados y el nuestro no parecen haber modificado la edad de experimentación con el tabaco. Este hecho sugiere la necesidad de intensificar los programas preventivos precoces, porque hay todavía pocos estudios ${ }^{4,8,12,13}$ que demuestran una tendencia descendente del consumo tabáquico, y porque las intensas campañas publicitarias de nuestro país podrían obstaculizar la tendencia decreciente, como ya sucedió en EEUU ${ }^{16}$. Tengamos en cuenta que en el Reino Unido ${ }^{14}$ la relación entre la inversión en programas preventivos del tabaquismo y la inversión en la publicidad y promoción del tabaco es $1 / 10$, y puede que en nuestro país la relación sea aún más desfavorable. Además, varios son los investigadores que demuestran que el objetivo prioritario de la publicidad del tabaco son los jóvenes ${ }^{14,16,33,38}$ y en ocasiones específicamente las jóvenes ${ }^{17,20,21}$. La densidad de anuncios en los alrededores de las escuelas primarias y secundarias, el uso de iconos atractivos para los adolescentes, como el de Camel, de productos juveniles, gorras, camisetas, etcétera, para soporte de marcas, y el desarrollo de espectáculos deportivos ligados a la publicidad del tabaco (o de sus marcas), que han sido reflejados por algunos autores ${ }^{39}$, también suceden en nuestro país. La revista oficial de Tabacale$\mathrm{ra}^{40.41}$ ratifica el uso de los productos citados y su vinculación al deporte de aventura, para promocionar las marcas de mayor aceptación entre los adolescentes y jóvenes, y recoge los valores del tabaco que la publicidad magnifica: la amistad, el erotismo y la relajación. Valores altamente apetecidos en una etapa de la vida con elevada socialización, intensa pulsión sexual y, a veces, desajuste psíquico.

De todos los determinantes del tabaquismo adolescente, bien estudiados en diversos países $^{19.20,21,27,28.34}$, nosotros sólo elegimos un factor personal (el fracaso escolar) y un factor social (el tabaquismo entre las personas con las que conviven los jóvenes y en sus profesores) para explorar su variabilidad en la población estudiada y ver si formaban parte del modelo. El análisis multivariante puso de manifiesto que, en el grupo $\mathrm{N}_{10-11}$, son las variables ambientales las que tienen un mayor peso en el modelo: sobre todo la accesibilidad al tabaco y al alcohol y la permanencia en discotecas y salas de juego. Para Froján ${ }^{42}$, los determinantes del inicio 
del consumo están ligados al proceso de socialización, que las citadas variables ambientales reflejan. En el grupo $\mathrm{N}_{13-14}$ las variables ambientales ocupan también los primeros puestos en orden de influencia, pero la ausencia injustificada a clase y el tabaquismo del profesorado forman así mismo parte del modelo, del que participa también la disponibilidad de dinero, la edad y el sexo. Los porcentajes de compra de tabaco para ambos grupos etarios $(2,8 \%$ y $20,2 \%)$ denuncian el incumplimiento de la norma legal en nuestra comunidad.

De nuestra investigación pueden deducirse algunas claves para la elaboración de programas extraescolares de prevención del tabaquismo adolescente, dirigidos a niños escolarizados en nuestro medio:

- Hay un escaso asociacionismo juvenil, por ello las actividades desarrolladas en clubes $\mathrm{y}$ asociaciones, que han realizado en lugares como el Reino Unido, Francia o Escandinavia ${ }^{34,43}$, no alcanzarían en nuestra comunidad grandes coberturas.

- En el hogar sería posible acceder a un gran número de adolescentes, a través de campañas publicitarias televisivas (de las que existe experiencia en EEUU ${ }^{21}$, y mediante información escrita personalizada, como han hecho algunos programas extraescolares ${ }^{43}$; incluso vertida en revistas y tebeos infanto-juveniles, que parecen tener enorme influencia sobre los adolescentes ${ }^{44}$. También la radio y todos los soportes de comunicación auditiva (discos, cintas, etc.) serían un buen vehículo de mensajes antitabaco con soporte musical, dado el interés de los adolescentes por la música, como actividad recreativa dentro del hogar.

- Los líderes juveniles en el campo del deporte podrían ser los mejores transmisores del mensaje, constatado el interćs que la actividad deportiva suscita entre la población estudiada, sea como practicantes o como observadores. El uso de personajes del mundo del deporte, la canción, la TV o el cine parece haber sido muy eficaz en otros países ${ }^{44}$, y podría ser también muy adecuado en nuestra comunidad.

- Todos los lugares destinados a la práctica deportiva (polideportivos, gimnasios, campos deportivos de cualquier especialidad) deberían utilizarse para la difusión de mensajes preventivos. Por su potencial cobertura, sería muy necesario restringir la publicidad y promoción del tabaco o de sus marcas en tales lugares. Por contra, un estudio catalán muestra que se fumaba en casi la mitad de los polideportivos sometidos a estudio en Sabadell ${ }^{45}$.

- En cuanto a la intervención ambiental, es poco realista pretender llevar la publicidad antitabaco a las discotecas y salas de juego, no muy frecuentadas en estos grupos etarios, teniendo en cuenta los intereses económicos de los propietarios de tales lugares. La más realista de las intervenciones ambientales sería controlar que no se venda tabaco a los menores de 16 años, como la ley ordena, norma transgredida ante más del $20 \%$ de los niños mayores. Y ello, especialmente en los comercios de chucherias y golosinas, donde venden cigarrillos por unidades, lo que aumenta la accesibilidad al tabaco de los niños, al hacerlos económicamente más asequibles, y en las máquinas expendedoras. La importancia de estos lugares como centros proveedores ha sido puesta de manifiesto en otros países ${ }^{43}$.

También parece importante suprimir la publicidad del tabaco en las vallas publicitarias exteriores, dado que el callejeo con familiares y amigos es una actividad muy practicada, que expone a los jóvenes masivamente a la influencia de dicha publicidad, y hay cvidencia científica de la especial sensibilidad de los jóvenes a la publicidad del tabaco $^{46}$.

Aprovechando que ir de compras es otra actividad muy frecuente, sobre todo en el grupo $\mathrm{N}_{10-11}$, que hace recados para el hogar familiar, los establecimientos que reciben a 
estos pequeños clientes podrían ser otro lugar donde se exhibiera publicidad preventiva del tabaquismo.

En conclusión: La prevención extraescolar del tabaquismo adolescente es un campo abierto a la investigación en nuestro país. De nuestro estudio parece deducirse que la información escrita y las campañas mediáticas por radio y $\mathrm{TV}$, realizadas por personajes del deporte y otros espectáculos, podrían conectar con los gustos e intereses de los jóvenes y tener amplia cobertura. No obstante, habría que continuar estudiando qué programas extraescolares son los más adaptados a las características de los adolescentes españoles, en las distintas comunidades autónomas, y evaluar cuáles de tales programas tienen un mayor impacto sobre el tabaquismo adolescente, y cuáles son más eficientes.

\section{AGRADECIMIENTOS}

A la Dirección Provincial del Ministerio de Educación y Ciencia en Asturias, y a los profesores y alumnos involucrados, por toda la colaboración prestada. Al Programa Europa contra el Cáncer, de la Unión Europea, que financió con 100.000 ECUS el programa colaborativo «Smoking Prevention and Children: Intervention in school and out of school», ref. SOC 94-202185, a las universidades de Birmingham (School of Education), Maastricht (Facultad of Health Sciences) y Oviedo (Facultad de Medicina).

\section{BIBLIOGRAFÍA}

1. Villalbí JR, Aubá J, García-González A. Evaluación de un proyecto de prevención primaria del tabaquismo: el proyecto piloto PASE de Barcelona. Rev San Hig Pública 1992; 66: 143-8.

2. Villalbí JR, Aubá J, García A. Resultados de un programa escolar de prevención del abuso de sustancias adictivas. Proyecto piloto PASE de Barcelona. Gac Sanit 1993; 7: 70-77.

3. Villalbi JR, Nebot M, Comín E, Murillo C. Consumo precoz de tabaco en escolares. Rev San Hig Pública 1990; 64: 613-23.
4. Vallescar R, Moreno V, Sarquella J, Vilar S, Martín M. Consumo de tabaco de la comarea de La Garrotxa. Estudio comparativo 1982-86. Gac Sanit 1991; 5: 125-9.

5. Muñoz MT, Plaza I, Madero R, López D, Otero J, Hidalgo I et al. Estudio de Fuenlabrada: Hábito tabáquico en niños y adolescentes. Influencia sobre las variables de riesgo cardiovascular. An Esp Pediatr 1989; 31:331-5.

6. Goicoechea JM, Panella J, Portella E. Características del tabaquismo en el medio escolar en Andorra. Gac Sanit 1992; 6: 122-7.

7. Aubá J, Villalbí JR. Tabaco y adolescentes: Influencia del entorno personal. Med Clin (Barc) 1993; 100: 506-9.

8. Salto E, Plans P, Fuentes M, Pardell H, Salleras L. Epidemiología del hábito tabáquico entre los escolares y jóvenes de Cataluña. An Esp Pediatr 1993; Suppl 55P: 146-8.

9. Onís M de, Villar J. La consommation de tabac chez la femme espagnole. Wld Hlth Statist Quart 1991; 44: 80-8.

10. López ML, Nájera P, López C, Secall L, Valle $\mathrm{MO}$, Cueto A. Adolescencia en riesgo; una encuesta de salud entre estudiantes de COU. Gac Sanit 1992; 6: 157-63.

11. Mendoza R, Sagrera MR, Batista JM. Conductas de los escolares españoles relacionadas con la salud (1986-90). Madrid: CSIC; 1994.

12. Vaqué J, Salleras L. Acciones antitabáquicas desarrolladas en Cataluña. Aten Primaria 1987; 4: 4-9.

13. Villalbí JR, Vives R, Nebot M, Díez E, Ballestín M. El tabaco y los escolares: contexto, opiniones y comportamiento. Med Clin (Barc) 1998; 110: $579-81$.

14. Foulds J, Godfrey Ch.Counting the costs of children's smoking. BMJ 1995; 311: 1152-4.

15. Smith Ch, Nutbeam D, Moore L, Roberts Ch, Catford J. Current changes in smoking altitudes and behaviours among adolescents in Wales, 1986-92. J Public Health Med 1994; 16: 165-71.

16. Anónimo. Trends in Smoking Initiation Among Adolescents and Young Adults - United States, 1980-89. MMWR 1995; 44: 521-24.

17. Swan AV, Melia RJW, Fitzsimons B, Breeze E, Murray M. Why do more girls than boys smoke cigarettes? Health Ed J 1989; 48: 59-64.

18. Nelson DE, Giovino GA, Shopland DR, Mowery PD, Mills SL, Eriksen MP. Trends in cigarette 
smoking among US adolescents, 1974-1991. Am J Public Health 1995; 85: 34-40.

19. Glynn TJ, Anderson M, Schwarz L. Tobacco-Use reduction annong high-risk youth: recommendations of a National Cancer Institute Expert Advisory Panel. Prev Med 1991; 20: 279-91.

20. Bellew B, Wayne D. Prevention of smoking among schoolchildren: a review of research and recommendations. Health Ed J 1991; 50: 3-8.

21. Stead M, Hastings G, Tudor-Smith Ch. Preventing adolescent smoking: a review of options. Health Ed J 1996; 55: 31-54.

22. CDC. Guidelines for school health programs to prevent tobacco use and addiction. MMWR 1994; 43: 1-18.

23. Bruvold WH. A meta-analysis of adolescent smoking prevention programs. Am J Public Health 1993; 83: 872-80.

24. Perry Ch, Killen J, Telch M, Slinkard LA, Danaher BG. Modifying smoking behaviour of teenagers: A School-Based intervention. Am J Public Health 1980; 70: 722-5.

25. Mc Alister A, Perry Ch, Killen J, Slinkard LA, Maccoby N. Pilot Study of Smoking, Alcohol and Drug Abuse Prevention. Am J Public Health $1980 ; 70: 719-21$.

26. Flay BR, Koepke D, Thomson SJ, Santi S, Allan J, Brown KS. Six-Year follow-up of the first Waterloo School Smoking Prevention Trial. Am J Public Health 1989; 79: 1371-6.

27. Fergusson DM, Lynskey MT, Horwood LJ. The role of peer affiliations, social, family and individual factors in continuities in cigarette smoking between childhood and adolescence. Addiction 1995; 90: 647-59.

28. Vries H, Dijkstra M. Non-smoking: your choice, a Dutch smoking prevention programme- A Case Study. En: James Ch, Balding J, Harris D Ed. World Yearbook of Education 1989. Health Education. New York: Kogan Page; 1990.p. 20-30.

29. Vries H, Kok GI. From determinants of smoking behaviour to the implications for a prevention programme. Health Fd Res 1986; 1: 85-94.

30. Vries H, Backbier E, Dijkstra M, Van Breukelen G, Parcel G, Kok G. A Dutch social influencie smoking prevention approach for vocacional school students. Health Ed Res 1994; 9: 365-74.

31. Aubá J, Villalbí JR. Prevención desde la escuela del uso de tabaco y de otras sustancias adictivas. Gac Sanit 1990; 4: 70-75.
32. Best $\mathrm{J} \Lambda$, Thomson SJ, Santi SM, Smith E $\Lambda$, Brown KS. Preventing cigarette smoking among school children. Ann Rev Public Health 1988; 9: 161-201.

33. Elders MJ, Perry Ch, Eriksen MP, Giovino GA. The report of surgeon general: preventing tobacco use among young people. AJPH 1994; 84: 543-7.

34. Vries H, Dijkstra M, Kok G. Young people and their smoking behaviour. En: Heller T, Bailey L, Pattison S, Ed. Preventing cancers. Londres: The Open University, 1992; 154-61.

35. Prochaska JO, Redding CA, Evers Ke. The transtheoretical model and stages of change. En: Glanz K, Lewis FC, Rimer BK, ed. Health behavior and health education (2." ed). San Francisco: Jossey-Bass Publishers; 1997.p. 60-84.

36. Brug J, Steenhuis IHM, Van Assema P, Vries H. The impact of a computer-tailored nutrition intervention. Prev Med 1996; 25: 236-42.

37. Mendoza R, Batista JM, Sánchez M. Carrasco AM. El consumo de tabaco, alcohol y otras drogas en los adolescentes escolarizados españoles. Gac Sanit 1998; 12: 263-71.

38. López ML, Nájera P, López C, Secall L, Valle O, Donate I, Cueto A. Publicidad, legislación publicitaria y salud. Gac Sanit 1992; 6: 220-4.

39. Kessler DA. Nicotine addiction in young people. New Eng J Med 1995; 333: 186-8.

40. Anónimo. Actualidad Tabaquera 1996; 510: 34-54.

41. Anónimo. Actualidad Tabaquera 1996; 506: 48.

42. Froján MX, Santacreu J. Modelo de génesis, adquisición, mantenimiento e insatisfacción con el hábito de fumar. Bol Psicol 1992; 34: 7-25.

43. Smith Ch. Smoking among young people: some recent developments in Wales. Health Ed J 1991; 50: 8-11.

44. Woods C. Anti-Smoking education: working with the teenage magazine market. Health Ed J 1991; 50: 19-22.

45. Serra C, Bonfill X, López V. Consumo y venta de tabaco en lugares públicos: evaluación del cumplimiento de la normativa vigente. Gac Sanit 1997; 11: 55-65.

46. Amos A. Young People, tobacco and 1992. Health Ed J 1991; 50: 26-30. 